\title{
Nonlinear Vibration of an Elastic Soft String: Large Amplitude and Large Curvature
}

\author{
De-Min Zhao, Shan-Peng Li, Yun Zhang, and Jian-Lin Liu \\ Department of Engineering Mechanics, College of Pipeline and Civil Engineering, China University of Petroleum (East China), \\ Qingdao 266580, China
}

Correspondence should be addressed to Jian-Lin Liu; liujianlin@upc.edu.cn

Received 12 April 2018; Accepted 7 June 2018; Published 22 July 2018

Academic Editor: Carlos Llopis-Albert

Copyright (C) 2018 De-Min Zhao et al. This is an open access article distributed under the Creative Commons Attribution License, which permits unrestricted use, distribution, and reproduction in any medium, provided the original work is properly cited.

\begin{abstract}
Mechanical nonlinear vibration of slender structures, such as beams, strings, rods, plates, and even shells occurs extensively in a variety of areas, spanning from aerospace, automobile, cranes, ships, offshore platforms, and bridges to MEMS/NEMS. In the present study, the nonlinear vibration of an elastic string with large amplitude and large curvature has been systematically investigated. Firstly, the mechanics model of the string undergoing strong geometric deformation is built based on the Hamilton principle. The nonlinear mode shape function was used to discretize the partial differential equation into ordinary differential equation. The modified complex normal form method (CNFM) and the finite difference scheme are used to calculate the critical parameters of the string vibration, including the time history diagram, configuration, total length, and fundamental frequency. It is shown that the calculation results from these two methods are close, which are different with those from the linear equation model. The numerical results are also validated by our experiment, and they take excellent agreement. These analyses may be helpful to engineer some soft materials and can also provide insight into the design of elementary structures in sensors, actuators and resonators, etc.
\end{abstract}

\section{Introduction}

Mechanical vibration of slender structures, such as beams, strings, rods, plates, and even shells, occurs widely in a variety of areas, spanning from aerospace, automobile, cranes, ships, offshore platforms, and bridges to MEMS/NEMS (micro/nanoelectromechanical system) [1-5]. On one hand, the huge vibration amplitude can shorten the lifetime of the whole structures, with the accumulation of damage or fatigue of materials [6]. On the other hand, the advantage of mechanical vibration can be successfully utilized in a lot of spectrums. For instance, at the nanoscale, Wang et al. used a situ transmission electron microscopy (TEM) to measure the dynamic deflection of a cantilever made of multiwalled carbon nanotube, which was excited to resonance in TEM $[7,8]$. Besides this, Zheng and Jiang proposed the concept of creation of nanomechanical systems of operating frequency up to several gigahertz, based on the oscillation of a core in a multiwall carbon nanotube [9].
The most intriguing issue on vibration is its nonlinear effect, as the strong nonlinearity can lead to many special phenomena. Up to now, the nonlinear vibration of structures has aroused extensive attention in the past decades. A central task of the nonlinear vibration of beams is to seek their nonlinear frequencies, and much effort has been devoted to this problem. For example, Hemmatnezhad and Ansari [10] studied the frequency of a functionally graded beam by means of a finite element formulation. In their model, the von Karman type nonlinear strain-displacement relationship is employed, and the effects of transverse shear deformation are included based upon the Timoshenko beam theory. Similarly, to further seek the nonlinear frequencies, Gunda and Gupta [11] investigated the vibration of a composite beam, Nikkar and Bagheri [12] explored the cantilever beam with an intermediate lumped mass, and Yu and Wu et al. [13] studied the beam with immovable spring-hinged ends. More related works include that Raju and Rao [14] formulated the nonlinear vibration of the beam using multiterm admissible 
functions for the first mode. Then He [15] used the variational approach to investigate the vibration frequency of a uniform cantilever beam carrying an intermediate lumped mass. Hoseini and Pirbodaghi et al. [16] presented the homotopy analysis method to study the accurate analytical solution for the nonlinear fundamental natural frequency of a tapered beam with large amplitude vibration.

At the micro/nanoscale, Gheshlaghi [17] developed the Euler-Bernoulli beam model and calculated the nonlinear natural frequencies of the first two modes of a nanowire. In succession, the harmonic balance method and the asymptotic numerical method were combined to solve the equations of a comprehensive multiphysics model of cantilever made of carbon nanotube [18]. Moreover, using the different model, i.e., the Eringen's nonlocal elasticity theory, Simsek [19] calculated the nonlinear vibration frequency of a nanobeam with axially immovable ends. Similarly, Nazemnezhad et al. [20] got the exact solution for the nonlinear vibration of a nanobeam, in use of the nonlocal Euler-Bernoulli theory. For the applications of devices, Feng et al. [1] studied the nonlinear vibration of a dielectric elastomer-based microbeam resonator, where the gas damping and excitation are considered. Han and Zhang [2] designed a doubly clamped microresonator based on the large amplitude vibration model. Furthermore, in the experiments, several measurement methods for the nonlinear vibration of slender beams were proposed [21, 22].

Although much work has been done on the nonlinear vibration of beams and in most of references this behavior was termed as "large amplitude," however, it should be stressed that only the normal strain with von Karman type was considered in most of works. In practice, when a beam vibrates with large displacement, its amplitude can even amount to the value on the same order as its length. That is to say, the large amplitude vibration of the string should be associated with large displacement, large rotation, and large curvature, which should be taken into consideration [23]. Pai and Nayfeh [24] studied the large deformation body especially considering the large deformation and rotation, which is a bit complex. Babilioa and Lenci [25, 26] gave definitions on mechanical and geometric curvatures and, similarly, Kopmaz and Gündogdu [27] presented different concepts on mathematical and physical curvatures. With the same idea, Semler et al. [28] developed a beam equation on the nonlinear vibration of a pipe conveying fluid. Zhao et al. [29] used the similar model to investigate the nonlinear vibration of a nanobeam with surface effects. In addition, Vlajic and Fitzgerald et al. [30] studied the prestressed beam with large variable curvature, and they provided the analytical formulation for static configurations, natural frequencies, and mode shapes, which were validated by the experiment and finite element method. It is clearly seen that the abovementioned bibliography mostly focuses on beams, and little literature on string vibration has been mentioned. Although Nayfeh and Mook [31] analyzed the nonlinear (free and forced) vibrations of strings and Benedettisi and Rega [32] studied the forced vibration of a suspended cable associated with quadratic and cubic nonlinearities, only small amplitude of the string is considered. The vibration characteristics of a light axially moving band were investigated by Koivurova in use of the Fourier-Galerkin-Newton method [33], where the von Karman strain was taken into account.

In the present study, we do not concentrate on the vibration of beams or plates but mainly on an elastic string or rod made of soft materials such as rubber materials, where large deformation and curvature can often happen when it vibrates. In this situation, the string will experience a very large displacement and especially a large curvature. This issue is not trivial, as the equation is associated with very strong nonlinearity. Although the complex normal form method (CNFM) developed by Nayfeh [34] was adopted to analyze the nonlinear vibration [29] it works very well in weak nonlinear vibration system. Then Leung et al. and Zhang et al. $[35,36]$ modified the traditional CNFM to predict the natural frequency in the strong nonlinear dynamics. For the CNFM introduced by Nayfeh [34], the fundamental frequency is independent of parameters of the nonlinear terms of the equation, which equals to the natural frequency of the derived linear system. However, for the modified CNFM [35, 36], the fundamental frequency is unknown and needs to be determined by the parameters of the nonlinear terms of the equation.

The outline of the paper is organized as follows. In Section 2, the dynamics equation of an elastic string with large amplitude and large mechanical curvature is derived based on the Hamilton principle. Next, in Section 3, the Galerkin method using the nonlinear mode shape function is used to discretize the partial differential equation to ordinary differential equation. Then, the modified complex normal CNFM is adopted to get the semianalytical solution of the strong nonlinear vibration of the string in Section 4 . In Section 5, the semianalytical results including the time history diagram, string configuration, total string length, and fundamental frequency are calculated, which are compared with those from the numerical computation and our selfdesigned experiment. Finally, the conclusion is given in Section 6. Although the analysis is aiming to investigate the string vibration, the route of line can be extended to explore the vibration of some other elastic structures, such as rods, beams, plates, and shells.

\section{Model Formulation}

We consider an elastic string, with an initial configuration of Line $O B$, as shown in Figure 1. Refer to the Cartesian coordinate system $O-\bar{x} \bar{y}$, where the origin is located at point $O$. The original length of the string is $L$, and its cross-section area is $A$. Young's modulus and mass density of the string are $E$ and $\rho$, respectively, and point $D_{0}$ is the midpoint of the string. When the string starts to vibrate in the $(\bar{x}, \bar{y})$ plane, its axis is elongated and the morphology is curvilinear, which is schematized in Figure 1. We examine two arbitrary adjacent material points $C_{0}(\bar{x}, 0)$ and $C_{0}^{\prime}(\bar{x}+\mathrm{d} \bar{x}, 0)$, both in $\bar{x}$-axis with infinitesimal distance $\mathrm{d} \bar{x}$ in the original configuration; then they transfer to the positions $C_{1}\left(\bar{x}_{1}, \bar{y}_{1}\right)$ and $C_{1}^{\prime}\left(\bar{x}_{2}, \bar{y}_{2}\right)$ after deformation. Considering the large deformation of the string, the curvilinear coordinate is introduced, where the arc length $\bar{s}$ is measured from the origin point $O$ along the axis of the string. 


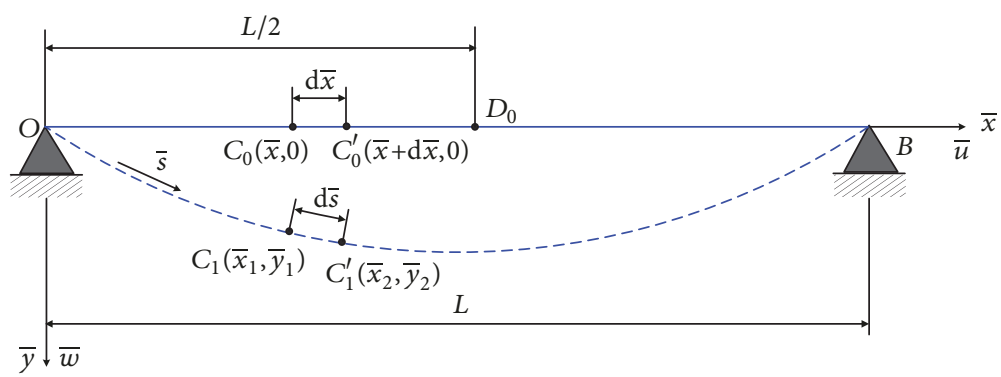

FIgURE 1: Schematic of the vibrational configuration of an elastic string with large deformation.

According to Figure 1, the geometric relations of the string are given as $[28,29,31]$

$$
\begin{aligned}
\bar{x}_{1}-\bar{x} & =\bar{u} \\
\bar{y}_{1} & =\bar{w} \\
\bar{x}_{2}-(\bar{x}+\mathrm{d} \bar{x}) & =\bar{u}+\mathrm{d} \bar{u} \\
\bar{y}_{2} & =\bar{w}+\mathrm{d} \bar{w},
\end{aligned}
$$

where $\bar{u}$ and $\bar{w}$ are the displacements of point $C_{0}$ along $\bar{x}$ and $\bar{y}$ directions, respectively. It can be seen that, before deformation, the microelement between $C_{0}$ and $C_{0}^{\prime}$ has the length $\mathrm{d} \bar{x}$ and it becomes $\mathrm{d} \bar{s}$ after deformation. As a result, the expression of $\mathrm{d} \bar{s}$ is derived as

$$
\mathrm{d} \bar{s}=\sqrt{\left(\bar{x}_{2}-\bar{x}_{1}\right)^{2}+\left(\bar{y}_{2}-\bar{y}_{1}\right)^{2}}=\sqrt{\left(1-\bar{u}^{\prime 2}\right)^{2}+\bar{w}^{\prime 2}},
$$

where $\bar{u}^{\prime}=\mathrm{d} \bar{u} / \mathrm{d} \bar{x}$ and $\bar{w}^{\prime}=\mathrm{d} \bar{w} / \mathrm{d} \bar{x}$. Due to the fact that the value of $\bar{u}$ is much smaller than that of $\bar{w}$, the contribution of $\bar{u}^{\prime}$ can be negligible in the vibration process. Therefore, the strain of an arbitrary point in the string is given by

$$
\varepsilon=\frac{\mathrm{d} \bar{s}-\mathrm{d} \bar{x}}{\mathrm{~d} \bar{x}}=\sqrt{1+\bar{w}^{\prime 2}}-1
$$

In order to obtain the governing equation of the string, we deal with this problem by way of energy principle. Firstly, the kinetic energy of the string can be written as

$$
T=\int_{0}^{L} \rho A \dot{\bar{w}}^{2} \mathrm{~d} \bar{x}
$$

where $\dot{\bar{w}}=\mathrm{d} \bar{w} / \mathrm{d} t$ and $t$ is the time variable.

The elastic strain energy is decomposed into two portions; i.e., the first contribution originates from the pretension force of the string $N_{0}$ and the second one comes from the elongation in the vibration process. Consequently, in a period from $t_{1}$ to $t_{2}$, the strain energy can be expressed as

$$
U=\frac{E A}{2} \int_{t_{1}}^{t_{2}} \int_{0}^{L}\left(\frac{N_{0}}{E A}+\varepsilon\right)^{2} \mathrm{~d} \bar{x} \mathrm{~d} t .
$$

Considering there is no work from the nonconservative forces, the application of Hamilton principle yields

$$
\delta \int_{t_{1}}^{t_{2}}(T-U) \mathrm{d} t=0 .
$$

Because we study the soft slender string, the mechanical curvature is sufficiently accurate and used more conveniently for the integration $[25,26]$. Therefore, in use of the principle of variation, the governing equation of the string in vibration with large amplitude and large mechanical curvature can be deduced as

$$
\begin{aligned}
& \rho A \ddot{\bar{w}}-\left(N_{0}-E A\right)\left(\frac{\bar{w}^{\prime \prime}}{\sqrt{1+\bar{w}^{\prime 2}}}-\frac{\bar{w}^{\prime 2} \bar{w}^{\prime \prime}}{\left(1+\bar{w}^{\prime 2}\right)^{3 / 2}}\right) \\
& -E A \bar{w}^{\prime \prime}=0 .
\end{aligned}
$$

The boundary conditions at two ends are

$$
\begin{aligned}
& \bar{w}(0, t)=0, \\
& \bar{w}(L, t)=0,
\end{aligned}
$$

where $\ddot{\bar{w}}=\mathrm{d}^{2} \bar{w} / \mathrm{d} t^{2}$ and $\bar{w}^{\prime \prime}=\mathrm{d}^{2} \bar{w} / \mathrm{d} \bar{x}^{2}$.

It can be seen that when the amplitude of the string is big enough, the term $\bar{w}^{\prime 2}$ cannot be ignored in (7), and thus the governing equation is more complicated, as it has a very great mechanical curvature. As is well known, the value of $\bar{w}^{\prime}$ is close to zero when the vibration amplitude is small. If this term is omitted, (7) can degenerate to the classical string vibration equation, whose amplitude and curvature are both small and the equation is linear:

$$
\ddot{\bar{w}}=\frac{N_{0}}{\rho A} \bar{w}^{\prime \prime} .
$$

For convenience, the following nondimensional quantities are defined as

$$
\begin{aligned}
& w=\frac{\bar{w}}{L}, \\
& x=\frac{\bar{x}}{L}, \\
& r=\frac{1}{L} \sqrt{\frac{N_{0}}{\rho A},} \\
& \omega=\pi r, \\
& \tau=\omega t,
\end{aligned}
$$




$$
\begin{aligned}
& \beta_{1}=\frac{\left(1-E A / N_{0}\right)}{\pi^{2}}, \\
& \beta_{2}=\frac{E A}{N_{0} \pi^{2}} .
\end{aligned}
$$

Accordingly, (7) can be recast as

$$
\ddot{w}=\frac{\beta_{1} w^{\prime \prime}}{\left(1+w^{\prime 2}\right)^{1 / 2}}-\frac{\beta_{1} w^{\prime 2} w^{\prime \prime}}{\left(1+w^{\prime 2}\right)^{3 / 2}}+\beta_{2} w^{\prime \prime},
$$

and this equation is termed as the dimensionless large amplitude vibration (LAV) equation throughout this study.

Then the boundary conditions and initial conditions are rewritten as

$$
\begin{aligned}
& w(0, \tau)=0, \\
& w(1, \tau)=0, \\
& w(x, 0)=a \sin (\pi x), \\
& \dot{w}(x, 0)=0 .
\end{aligned}
$$

At the same time, the linear equation is nondimensionalized as

$$
\ddot{w}=\frac{1}{\pi^{2}} w^{\prime \prime}
$$

In the above equations, the related expressions are $\ddot{w}=$ $\mathrm{d}^{2} w / \mathrm{d} \tau^{2}, w^{\prime}=\mathrm{d} w / \mathrm{d} x$, and $w^{\prime \prime}=\mathrm{d}^{2} w / \mathrm{d} x^{2}$, and $a$ is the amplitude of the middle point in the string, i.e., point $D_{0}$.

\section{Mode Discretization with Exact Mode Shape}

The following task is to solve (11), which is an intractable problem. Clearly, it seems impossible to directly get the analytical solution of this highly nonlinear equation. Therefore, in use of the Taylor series, (11) can be expanded into the following polynomial expansion until 5 th-order terms, where the terms of higher order are ignored:

$$
\ddot{w}=\frac{w^{\prime \prime}}{\pi^{2}}+\beta_{1}\left(-\frac{3 w^{\prime 2}}{2}+\frac{15 w^{\prime 4}}{8}\right) w^{\prime \prime} .
$$

It is clear that (14) is still a high order and nonlinear partial differential equation (PDE), and seeking the closed form solution is not at hand. Herein, the Galerkin discretization method is utilized to transform the PDE into the ordinary differential equation (ODE). The previous result [18] tells us that the first-mode analysis of vibration is sufficient to capture the main nonlinear characteristics and can get the fundamental frequency accurately enough. Hence, we take the trial to only consider the first mode of the vibration, aiming to get an approximate solution for the first step. Moreover, the mode shape is a critical factor to affect the vibration of the continuously beam, rod, and string. For the small amplitude vibration of string with simply supported condition, the firstmode shape function is often assumed by linear mode shape function [31] described by $\sin (\pi x)$. However, for the large curvature vibration, especially the amplitude of the middle point nearly 0.4-0.5 times of span of simply supported string, the mode shape function, $\sin (\pi x)$, based on the linear the mode shape, fails. The exact mode shape function should be selected considering the physical experiments given in Section 5.3. Assume that the displacement $w(x, \tau)$ can be described as

$$
w(x, \tau)=q(\tau) W(x)
$$

and

$$
W(x)=\sin (\pi x) \sqrt{1+\cos ^{2}(\pi x)} .
$$

Substituting (15) and (16) into (14), multiplying both sides of the equation by $W(x)$ and then integrating both sides from 0 to 1 yields the following ODE equation with strong nonlinearities:

$$
\ddot{q}+\omega_{0}^{2} q+k_{1} q^{3}+k_{2} q^{5}=0,
$$

where $\omega_{0}^{2}=1.0745, k_{1}=-8.1836 \beta_{1}$, and $k_{2}=-53.5149 \beta_{1}$.

In order to compare the large curvature vibration with the small amplitude vibration, for the linear equation in (13), the classic mode shape function $\sin (\pi x)$ is adopted; thus the classic the dimensionless ODE can degenerate to $\ddot{q}+$ $q=0$. Then one can obtain the theoretical solution easily, which is named as theoretical solution of the linear equation throughout the paper.

\section{Semianalytical Solution Using CNFM Method}

Equation (17) is strong nonlinear system due to the nonlinear terms $q^{3}$ and $q^{5}$ which are far more large than the linear term q. Although He's variational method $[6,12,15,19]$, harmonic balance method [18], and Homotopy analysis method [16] are used widely in the strong nonlinear system, the modified CNFM method [29, 34-36] gives more accurate results based on the experience of the authors. Next, we use the modified CNFM approach to solve (17). We assume that the solution of (17) can be formulated as

$$
\begin{aligned}
& q=\xi+\bar{\xi} \\
& \dot{q}=i \omega_{1}(\xi-\bar{\xi}),
\end{aligned}
$$

where $\bar{\xi}$ is the complex conjugate of $\xi$ and $\omega_{1}$ is the unknown fundamental natural frequency to be determined. Introduce a nonlinear transformation from $\xi$ to $\eta$ in the form of

$$
\xi=\eta+h(\eta, \bar{\eta})
$$

where $\eta$ and $\bar{\eta}$ are complex conjugates and they are both complex functions.

The near identity transformation function $h$ including the variables $\eta$ and $\bar{\eta}$ is expressed as

$$
\begin{aligned}
h(\eta, \bar{\eta})= & \Delta_{1} \eta+\Delta_{2} \bar{\eta}+\Delta_{3} \eta^{3}+\Delta_{4} \eta^{2} \bar{\eta}+\Delta_{5} \eta^{2} \\
& +\Delta_{6} \bar{\eta}^{3}+\Delta_{7} \eta^{5}+\Delta_{8} \eta^{4} \bar{\eta}+\Delta_{9} \eta^{3} \bar{\eta}^{2} \\
& +\Delta_{10} \eta^{2} \bar{\eta}^{3}+\Delta_{11} \eta \bar{\eta}^{4}+\Delta_{12} \eta^{5}
\end{aligned}
$$

where $\Delta_{i}(i=1 . .12)$ are real numbers. 
Substituting (18)-(20) into (17), one can get

$$
\begin{aligned}
\dot{\eta}= & i \omega_{1} \eta+i \omega_{1} h-\frac{\partial h}{\partial \eta} \dot{\eta}-\frac{\partial h}{\partial \bar{\eta}} \dot{\bar{\eta}}+\frac{i \omega_{1}}{2}\left(\frac{\omega_{0}^{2}}{\omega_{1}^{2}}-1\right) \\
& \cdot(\eta+\bar{\eta}+h+\bar{h}) \\
& +\frac{i}{2 \omega_{1}}\left[k_{1}(\eta+\bar{\eta}+h+\bar{h})^{3}+k_{2}(\eta+\bar{\eta}+h+\bar{h})^{5}\right],
\end{aligned}
$$

where $h$ and $\bar{h}$ are complex conjugates. Substituting (19) and (20) into (18) leads to

$$
\begin{aligned}
q= & \eta+\bar{\eta}+\left(\Delta_{1}+\Delta_{2}\right)(\eta+\bar{\eta})+\left(\Delta_{3}+\Delta_{6}\right)\left(\eta^{3}+\bar{\eta}^{3}\right) \\
& +\left(\Delta_{4}+\Delta_{5}\right)\left(\eta^{2} \bar{\eta}+\eta \bar{\eta}^{2}\right)+\left(\Delta_{7}+\Delta_{12}\right)\left(\eta^{5}+\bar{\eta}^{5}\right) \\
& +\left(\Delta_{8}+\Delta_{11}\right)\left(\eta^{4} \bar{\eta}+\eta \bar{\eta}^{4}\right) \\
& +\left(\Delta_{9}+\Delta_{10}\right)\left(\eta^{3} \bar{\eta}^{2}+\eta^{2} \bar{\eta}^{3}\right) .
\end{aligned}
$$

To eliminate the secular terms $\eta, \eta^{2} \bar{\eta}$, and $\eta^{3} \bar{\eta}^{2}$ in (22), the following conditions should be met:

$$
\begin{gathered}
\Delta_{1}+\Delta_{2}=0 \\
\Delta_{4}+\Delta_{5}=0 \\
\Delta_{9}+\Delta_{10}=0 .
\end{gathered}
$$

Moreover, let the coefficients of terms $\bar{\eta}, \eta^{3}, \eta \bar{\eta}^{2}, \bar{\eta}^{3}, \eta^{5}, \eta^{4} \bar{\eta}$, $\eta^{2} \bar{\eta}^{3}, \eta \bar{\eta}^{4}$, and $\bar{\eta}^{5}$ in the right side of (22) vanish, as they are the nonresonant terms, which are much smaller than term $\eta$. Thus twelve equations are obtained in Appendix. Solving (23)-(25) and the related equations in Appendix, we can acquire the parameters $\Delta_{i}(i=1, \ldots, 12)$, which are given in Appendix.

After these operations, the resonant terms $\eta, \eta^{2} \bar{\eta}$, and $\eta^{3} \bar{\eta}^{2}$ will remain in (21), and the equation is simplified to

$$
\begin{aligned}
\dot{\eta}= & i \frac{1+\omega_{1}^{2}}{2 \omega_{1}} \eta+i \frac{3 k_{1}}{2 \omega_{1}} \eta^{2} \bar{\eta} \\
& +i \frac{1}{2 \omega_{1}}\left(10 k_{2}-3 k_{1} \Delta_{6}\right) \eta^{3} \bar{\eta}^{2},
\end{aligned}
$$

where the real parameters $\Delta_{6}$ is given in Appendix.

Assume

$$
\eta=\frac{1}{2} a e^{i \omega_{1} t}
$$

where $a$ is the dimensionless vibration amplitude and $\omega_{1}$ is the fundamental frequency which is to be determined. Substituting (27) into (26) leads to

$$
\begin{aligned}
\dot{a} & =0 \\
\omega_{1} & =\frac{\omega_{0}^{2}+\omega_{1}^{2}}{2 \omega_{1}}+\frac{3 k_{1}}{8 \omega_{1}} a^{2}+\frac{10 k_{2}-3 k_{1} \Delta_{6}}{32 \omega_{1}} a^{4} .
\end{aligned}
$$

The first equation in (28) indicates that the amplitude $a$ is a constant determined by the initial condition, and the second equation determines the fundamental frequency $\omega_{1}$. Substituting $\Delta_{i}(i=1, \ldots, 12), a$ and $\omega_{1}$ into (22), one can get the final solution of (17) as

$$
\begin{aligned}
q= & a \cos \left(\omega_{1} t\right) \\
+ & {\left[\frac{1}{4}\left(\Delta_{3}+\Delta_{6}\right) a^{3}+\frac{1}{16}\left(\Delta_{8}+\Delta_{11}\right) a^{5}\right] } \\
& \cdot \cos \left(3 \omega_{1} t\right)+\frac{1}{16}\left(\Delta_{7}+\Delta_{12}\right) a^{5} \cos \left(5 \omega_{1} t\right) .
\end{aligned}
$$

\section{Results and Discussion}

5.1. Numerical Scheme. To get a more accurate solution, the finite difference method (FDM) is used to directly calculate the PDE, which manifests a robust method. The central difference is adopted, i.e., $w^{\prime}=\left(w_{j+1}^{n}-w_{j-1}^{n}\right) / 2(\Delta x)$, $w^{\prime \prime}=\left(w_{j+1}^{n}-2 w_{j}^{n}+w_{j-1}^{n}\right) /(\Delta x)^{2}$, and $\ddot{w}=\left(w_{j}^{n+1}-\right.$ $\left.2 w_{j}^{n}+w_{j}^{n-1}\right) /(\Delta \tau)^{2}$, where $j$ and $n$ are both integers and $\Delta x$ and $\Delta \tau$ are the space and time steps, respectively. In the simulation process, the string is made of soft material such as rubber. The physical parameters of the string are measured as follows: mass density $\rho=798 \mathrm{~kg} / \mathrm{m}^{3}$, length $L=68.77 \mathrm{~mm}$, cross-section area $A=2.282 \mathrm{~mm}^{2}$, pretension force along the axial direction $N_{0}=0.68 \mathrm{~N}$, and Young's modulus $E=0.85$ $\mathrm{MPa}$. The computational program is written in MATLAB 12, where the time step $\Delta \tau$ is set as 0.001 and the space step $\Delta x$ is set as 0.01. In the FDM discretization, when $\tau=0$ and $\tau=\Delta \tau$, the displacement $\omega_{j}^{i}=a \sin [\pi(\Delta x)(j-1)],(i=1,2)$, and $j$ takes the value from 1 to 101 .

5.2. Time History Diagram of the Midpoint. Evidently, the midpoint $D_{0}$ is a critical point which should be carefully examined, as it may be associated with the largest amplitude of the string. When the initial amplitude of $D_{0}$ is bigger, such as $a=0.4$ and 0.45 , the related curves in the time history diagram are displayed in Figure 2. It can be seen that, with the evolution of time, the difference between the LAV model and the linear equation becomes more obvious; and the result from the semianalytical solution is in agreement with the numerical result, although there is slight difference as shown in Figure 2. As displayed in Figure 2(a), in the time interval from 0 to 20 , there are nearly 4 periods in the LAV model and only 3 periods in the linear equation model. It can also be observed when $\tau=15.7$ that the motion of the point predicted by the LAV equation is nearly one-half period faster than that by the linear equation. This indicates that the whole motion of the string predicted by the LAV equation is quicker than that predicted by the linear equation.

5.3. Vibrational Configurations. In what follows, we compute the configurations of the string with large amplitude $a=0.4$ in one period, which are shown in Figures 3(a)-3(h). Firstly, it is noted that there is great difference between the results from the LAV model and the linear equation model as shown in Figure 3. This again stresses that the linear equation is 


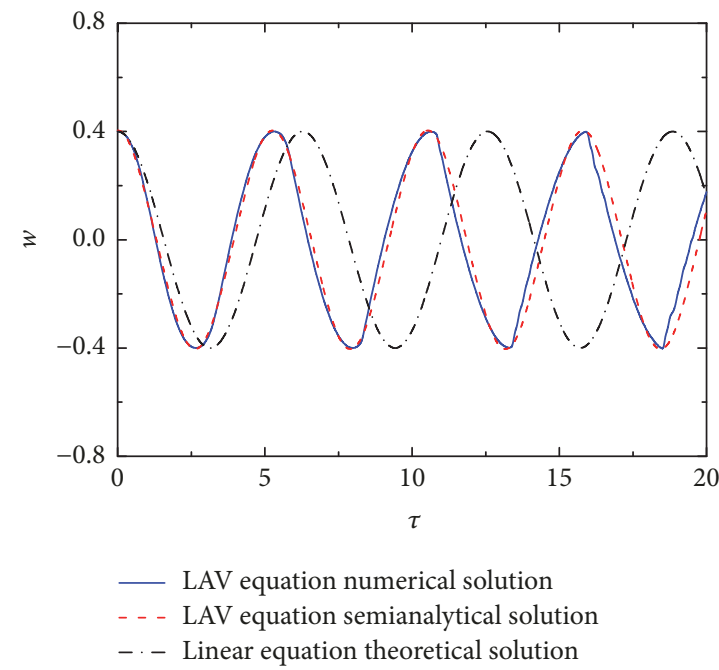

(a)

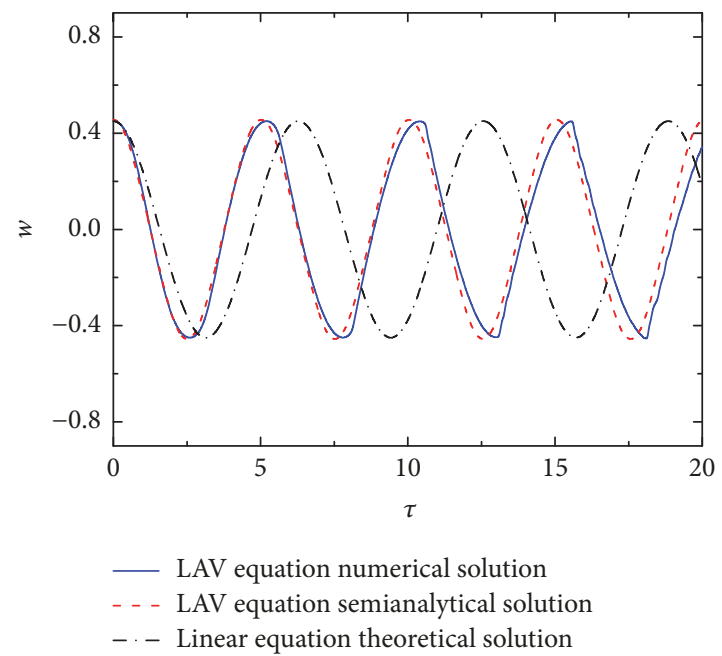

(b)

FIgURE 2: Time history diagram of point $D_{0}$, with the initial amplitudes (a) $a=0.4$ and (b) $a=0.45$.

insufficient to depict the vibration with large amplitude and large curvature. However, in the whole period, although there is difference between the results from the semianalytical method and numerical simulation, the configurations predicted by the two methods are close. Therefore, it should be mentioned that the calculated string morphologies by the semianalytical are more similar to those from the numerical results than those from the linear equation model.

The next phenomenon is that the numerical morphologies of the string are very complicated in the vibration process. For instance, when $\tau=5.3,6.0,8.0$, and 10.5 in Figures $3(a), 3(b), 3(d)$, and $3(h)$, there are some nearly horizontal segments appearing in the curves. This strange behavior may be attributed to the complex nonlinear dynamics response, where the clamped ends exert strong constraints on the string and vice versa; near the middle part of the string, the constraint is much weaker. Another possible reason is that the first-mode analysis of the string may be an oversimplified model and the higher-mode behaviors must be further considered to illustrate this phenomenon. Furthermore our semianalytical solution can also have the similar shapes in this figures, especially near the middle part of the string.

To verify this numerical result, we perform an experiment as a comparison, where the string configurations in the vibration are shown in Figures 4(a)-4(h). A rubber string with $1.408 \times 1.630 \mathrm{~mm}^{2}$ cross-section is fixed horizontally on a desktop at room temperature, whose original length $L$ is 50.85 $\mathrm{mm}$. To add the pretension force on the string, the deformed length of the string is elongated to $l=68.77 \mathrm{~mm}$ and origin area of the cross-section is $2.282 \mathrm{~mm}^{2}$. Thus the prestrain can be given as $\varepsilon=(l-L) / L=0.3524$, and using the measured value of Young's modulus $E=0.85 \mathrm{MPa}$, the pretension force is $N_{0}=0.68 \mathrm{~N}$. At first, in the vertical plane the midpoint of the string is excited by an original amplitude, which is 0.4 times of the string length $L$. Next, the string is released, and then its vibration sequences are recorded by the high speed camera (Phantom v2512 with 10000 frames per second). As shown in Figures 4(a), 4(b), 4(c), and 4(d), we find that there are really platform segments appearing in the string and the total configurations in these situations are close to the shape of trapezoid. Meanwhile, the string is no longer a straight line when it approaches the equilibrium position as shown in Figures 4(e) and 4(f), implying that the length of the string is bigger than its original length. These behaviors are beyond our imagination, as they demonstrate the complexity of the nonlinear dynamics.

5.4. Total String Length. It can be seen that in both the LAV equation and the linear equation, the extensibility of the string is considered; thus its length can be calculated and the two curves are compared in Figure 5. The nondimensional length of the string after deformation is expressed as $l / L=\int_{0}^{L} \sqrt{1+\bar{w}^{2}} \mathrm{~d} \bar{x} / L$. For the linear equation model, this nondimensional length is a periodic function with respect to the time $\tau$, i.e., $l / L(\tau+T / 2)=l / L(\tau)$. This means that the maximum and minimum values of the string length do not alter in the vibration process. Especially, when the string approaches the $x$-axis, it is really a straight line, and thus the value of $l / L$ is of the smallest value, i.e., one. This feature reemphasizes that the small amplitude vibration has linear properties. On the contrary, the oscillation behavior of the string length is more complex in the LAV model, where its minimum value increases with the elapsing time. In this situation, the dimensionless string length is always bigger than one, indicating that the string is always in the elongation state, even when it approaches the $x$-axis. In the experiment, such as in Figures 4(e), 4(f), 3(e), and 3(f), the shapes of the string are not merely straight lines and its length at this critical time must be larger than one. Evidently, this consistence on the experimental phenomenon can validate the efficiency of our numerical method. 


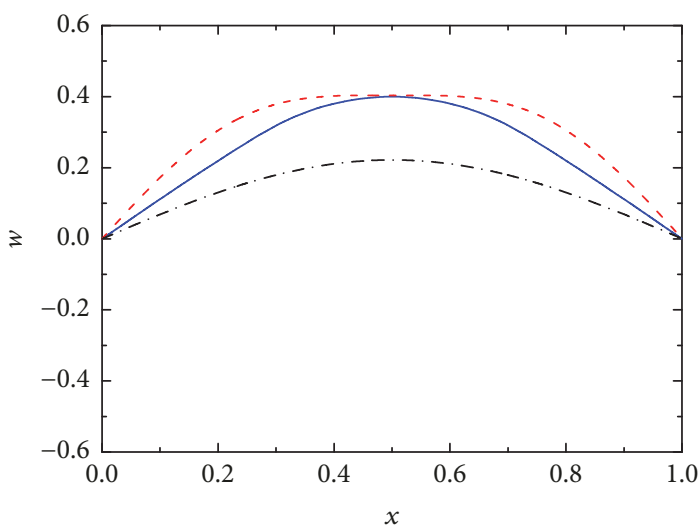

_ LAV equation numerical solution

- - - LAV equation semianalytical solution

-. - Linear equation theoretical solution

(a)

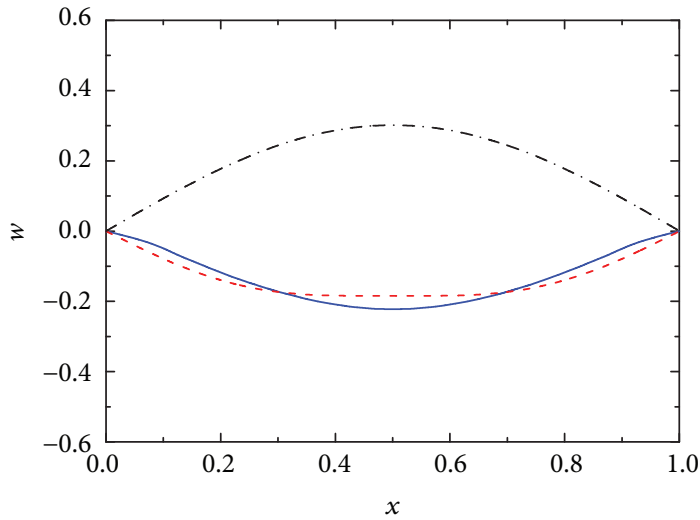

_ LAV equation numerical solution

- . - LAV equation semianalytical solution

-. - Linear equation theoretical solution

(c)

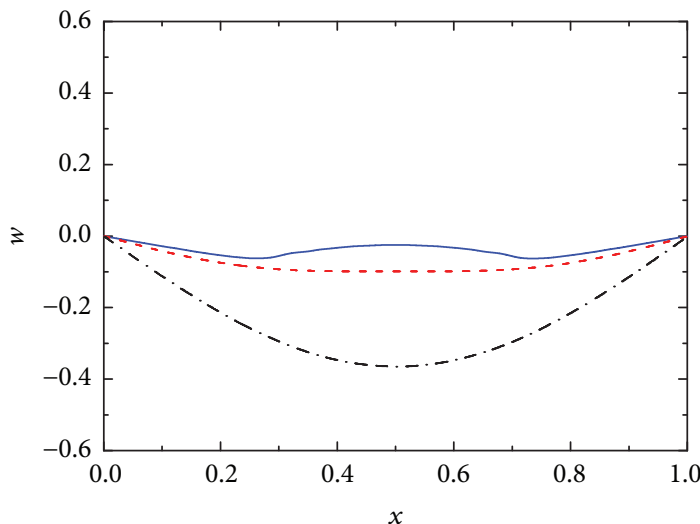

_ LAV equation numerical solution

- . - LAV equation semianalytical solution

-. - Linear equation theoretical solution

(e)

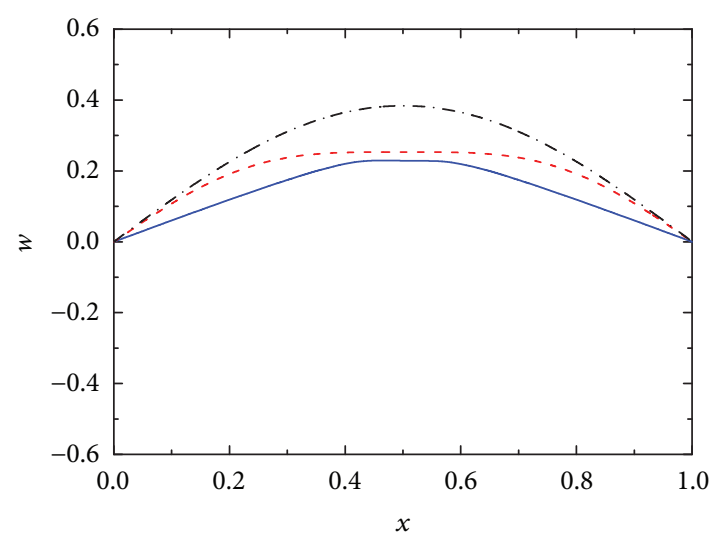

_ LAV equation numerical solution

- - - LAV equation semianalytical solution

-. - Linear equation theoretical solution

(b)

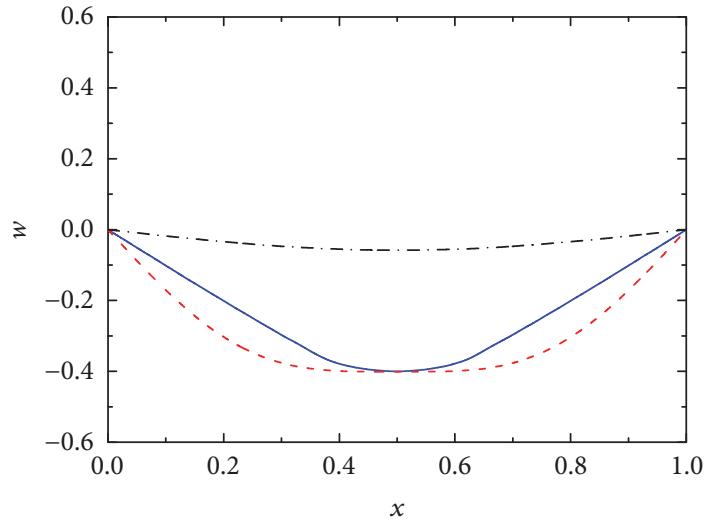

_ LAV equation numerical solution

- - - LAV equation semianalytical solution

- - - Linear equation theoretical solution

(d)

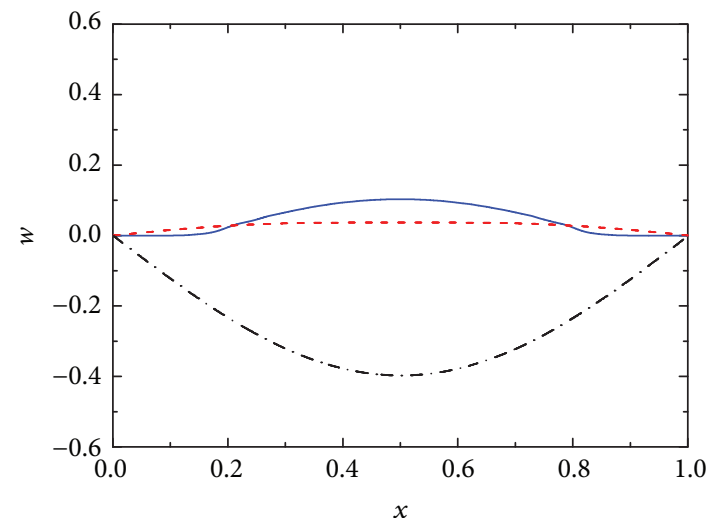

LAV equation numerical solution

- - LAV equation semianalytical solution

_. - Linear equation theoretical solution

Figure 3: Continued. 


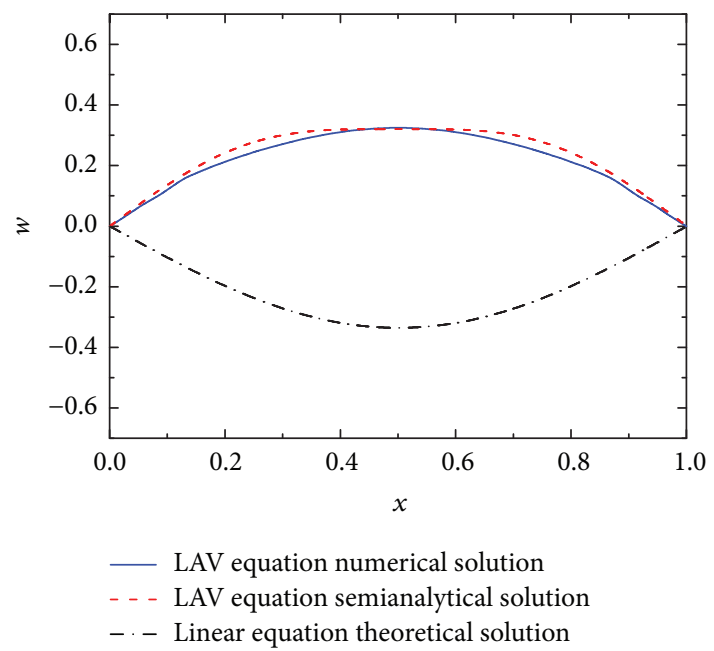

(g)

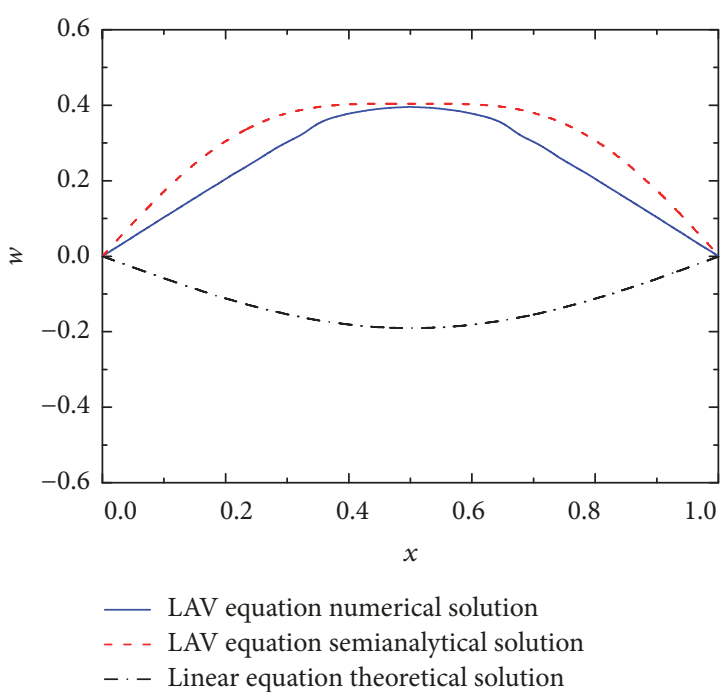

(h)

FIGURE 3: Vibrational configurations of an elastic string, when (a) $\tau=5.3$; (b) $\tau=6.0$; (c) $\tau=7.0$; (d) $\tau=8.0$; (e) $\tau=9.0$; (f) $\tau=9.3$; (g) $\tau=10$; (h) $\tau=10.5$.

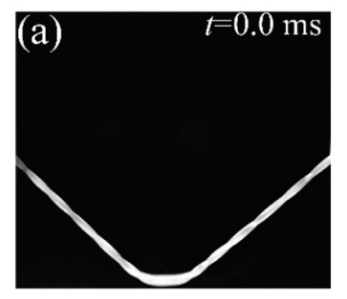

(a)

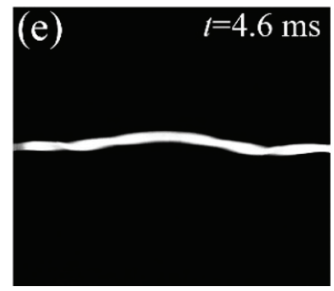

(e)

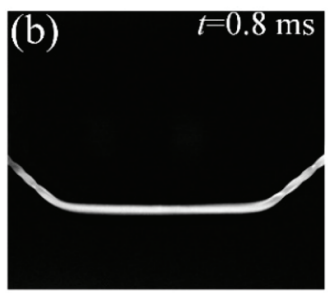

(b)

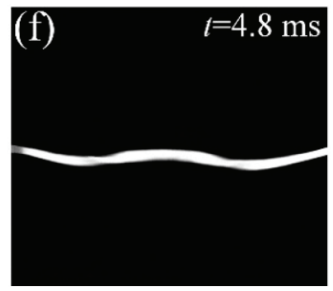

(f)

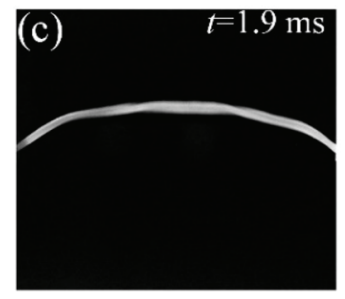

(c)

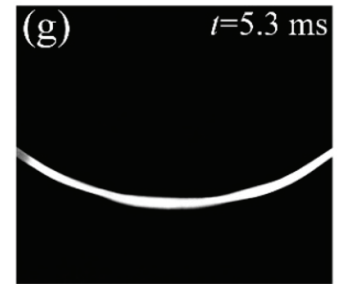

(g)

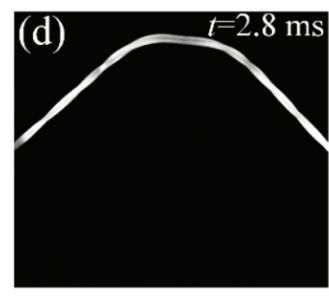

(d)

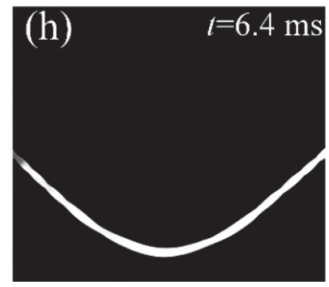

(h)

FIGURE 4: Vibrational configurations of the string in the experiment.

5.5. Fundamental Frequency. Another vibration characteristic parameter about vibration is the frequency, and in the current study, the value of the fundamental frequency is obtained by the solution of (28), and it is also tested by the experimental measurement. When the vibration has a small amplitude, vibration of the string, its dynamic behavior can be formulated by the linear equation $\ddot{q}+q=0$, with the natural frequency always being $\omega_{1}=1$, which is irrelative to the initial amplitude. We observed in Figure 6 the difference between the semianalytical method and experiment method is great. The main reason is that, for the small amplitude, the mode shape function should be $\sin (\pi x)$ rather than $\sin (\pi x) \sqrt{1+\cos ^{2}(\pi x)}$. The frequency exhibited in Figure 6 demonstrates that our mode shape function should not be used for the small amplitude vibration. However, for the large amplitude vibration, the fundamental frequency is not a constant and its value is greatly affected by the initial amplitude. As shown in Figure 6, with the increase of the amplitude, the fundamental frequency also increases, which is verified by the experimental results. Although there exits value difference between the semianalytical solution and the experimental data, their tendencies are the same and both of the two results are bigger than one with the increase of initial amplitude $a$. This behavior again demonstrates the complicated response of the nonlinear dynamics, which is very sensitive to the initial value. It should be clarified that our mode shape function only for the large amplitude vibration with the ratio (amplitude of the middle part of the string 


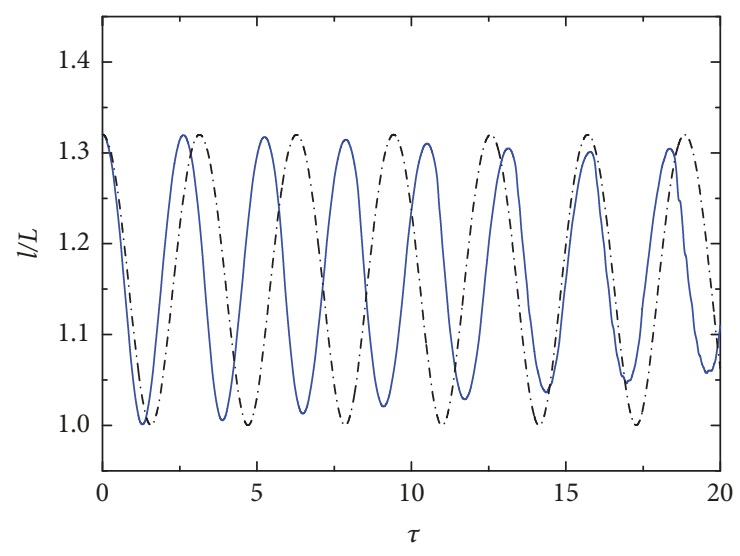

— LAV equation numerical solution

-.... Linear equation theoretical solution

FIGURE 5: Total length of the string based on the LAV equation and linear equation.

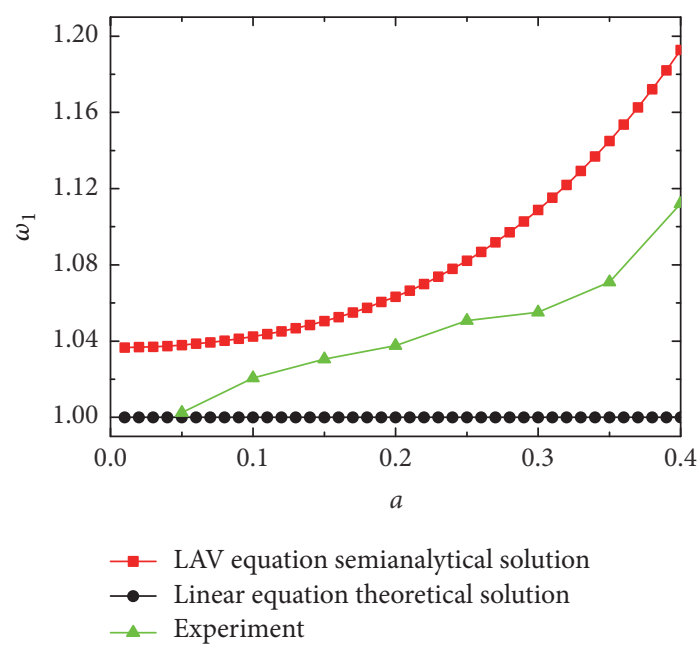

FIGURE 6: Relation between the fundamental $\omega_{1}$ and the initial amplitude $a$.

to the whole span) is nearly $0.2-0.5$. If the ratio is greater, different mode shape functions should be selected and the similar analysis process can be carried out.

\section{Conclusion}

In conclusion, the nonlinear vibration of a soft elastic string with large amplitude and large curvature has been systematically investigated in this study. The mathematical model in the absence of damping is developed based on the Hamilton principle. The exact mode shape function different from the counterpart derived from the linear equation was selected based on the experiment. For the large amplitude vibration, the time history diagram from the semianalytical is very close to that from the numerical result and these two results are very different from that of the linear model. The vibration configurations of the elastic string have the similar laws as those of the time history diagrams based on the comparison of the three models. We find that, in the numerical calculations, there are platform segments with the shape of trapezoid appearing in the configuration curves, and this phenomenon has been verified by our semianalytical solution and the experiment. The total length of the string in the large deformation shows that it is not a periodic function with respect to the time, which is distinct with the result of the linear model. We also point out that the fundamental frequency of the string with large amplitude vibration is greatly affected by the initial amplitude, which has the same tendency as our experiment result.

It should be mentioned that this study is only the first trial on the nonlinear vibration of elastic structures with large amplitude and large curvatures. The following work is expected, which will be extended to some other engineering structures, such as beams, rods, plates, and shells. These analyses may be beneficial to engineer some soft materials and can also shed light on the design of elementary structures in sensors, actuators and resonators, etc.

\section{Appendix}

\section{A. Equations to Determine Coefficients of Nonresonant Terms}

Besides (23)-(25), the other equations to determine the value of $\Delta_{i}(i=1, \ldots, 12)$ are

$$
\begin{aligned}
& 2 i \omega_{1} \Delta_{2}+\frac{i\left(\omega_{0}^{2}-\omega_{1}^{2}\right)}{2 \omega_{1}}=0 ; \\
& 2 i \omega_{1} \Delta_{3}-\frac{i\left(\omega_{0}^{2}-\omega_{1}^{2}\right)}{2 \omega_{1}}\left(\Delta_{3}+\Delta_{6}\right)-\frac{i k_{1}}{2 \omega_{1}}=0 ; \\
& 2 i \omega_{1} \Delta_{5}+\frac{3 i k_{1}}{2 \omega_{1}}=0 ; \\
& 4 i \omega_{1} \Delta_{6}+\frac{i\left(\omega_{0}^{2}-\omega_{1}^{2}\right)}{2 \omega_{1}}\left(\Delta_{3}+\Delta_{6}\right)+\frac{i k_{1}}{2 \omega_{1}}=0 ; \\
& 4 i \omega_{1} \Delta_{7}-\frac{i\left(\omega_{0}^{2}-\omega_{1}^{2}\right)}{2 \omega_{1}}\left(\Delta_{7}+\Delta_{12}\right)-\frac{3 i k_{1}}{2 \omega_{1}}\left(\Delta_{3}+\Delta_{6}\right) \\
& -\frac{i k_{2}}{2 \omega_{1}}=0 ; \\
& 2 i \omega_{1} \Delta_{8}-\frac{i\left(\omega_{0}^{2}-\omega_{1}^{2}\right)}{2 \omega_{1}}\left(\Delta_{8}+\Delta_{11}\right)-\frac{6 i k_{1}}{2 \omega_{1}}\left(\Delta_{3}+\Delta_{6}\right) \\
& -\frac{5 i k_{2}}{2 \omega_{1}}=0 ; \\
& 2 i \omega_{1} \Delta_{10}+\frac{3 i k_{1}}{2 \omega_{1}}\left(\Delta_{3}+\Delta_{6}\right)+\frac{10 i k_{2}}{2 \omega_{1}}=0 ; \\
& 4 i \omega_{1} \Delta_{11}+\frac{i\left(\omega_{0}^{2}-\omega_{1}^{2}\right)}{2 \omega_{1}}\left(\Delta_{8}+\Delta_{11}\right) \\
& +\frac{6 i k_{1}}{2 \omega_{1}}\left(\Delta_{3}+\Delta_{6}\right)+\frac{5 i k_{2}}{2 \omega_{1}}=0 ;
\end{aligned}
$$




$$
\begin{gathered}
6 i \omega_{1} \Delta_{12}+\frac{i\left(\omega_{0}^{2}-\omega_{1}^{2}\right)}{2 \omega_{1}}\left(\Delta_{7}+\Delta_{12}\right) \\
+\frac{3 i k_{1}}{2 \omega_{1}}\left(\Delta_{3}+\Delta_{6}\right)+\frac{i k_{2}}{2 \omega_{1}}=0 .
\end{gathered}
$$

\section{B. Formulas of Parameters $\Delta_{i}$}

The parameters $\Delta_{i}(i=1, \ldots, 12)$ are

$$
\begin{aligned}
& \Delta_{1}=-\Delta_{2}, \\
& \Delta_{2}=\frac{\omega_{1}^{2}-\omega_{0}^{2}}{4 \omega_{1}^{2}}, \\
& \Delta_{3}=-2 \Delta_{6}, \\
& \Delta_{6}=\frac{k_{1}}{\omega_{0}^{2}-9 \omega_{1}^{2}} \\
& \Delta_{4}=-\Delta_{5}, \\
& \Delta_{5}=-\frac{3 k_{1}}{4 \omega_{1}^{2}} \\
& \Delta_{7}=-\frac{3}{2} \Delta_{12}, \\
& \Delta_{12}=\frac{6 k_{1} \Delta_{6}-2 k_{2}}{25 \omega_{1}^{2}-\omega_{0}^{2}} \\
& \Delta_{8}=-2 \Delta_{11}, \\
& \Delta_{10}=\frac{3 k_{1} \Delta_{6}-10 k_{2}}{4 \omega_{1}^{2}} . \\
& \Delta_{11}=\frac{6 k_{1} \Delta_{6}-5 k_{2}}{9 \omega_{1}^{2}-\omega_{0}^{2}} \\
& \Delta_{10}
\end{aligned}
$$

\section{Data Availability}

No data were used to support this study.

\section{Ethical Approval}

Ethical approval was obtained for this study.

\section{Conflicts of Interest}

The authors declare that they have no conflicts of interest.

\section{Acknowledgments}

This study was supported by the National Natural Science Foundation of China (nos. 11672334, 11672335 and 11611530541) and the Endeavour Australia Cheung Kong Research Fellowship Scholarship from the Australian government.

\section{References}

[1] C. Feng, L. Yu, and W. Zhang, "Dynamic analysis of a dielectric elastomer-based microbeam resonator with large vibration amplitude," International Journal of Non-Linear Mechanics, vol. 65, pp. 63-68, 2014.

[2] J. Han, Q. Zhang, and W. Wang, "Design considerations on large amplitude vibration of a doubly clamped microresonator with two symmetrically located electrodes," Communications in Nonlinear Science and Numerical Simulation, vol. 22, no. 1-3, pp. 492-510, 2015.

[3] T. Beléndez, C. Neipp, and A. Beléndez, "Large and small deflections of a cantilever beam," European Journal of Physics, vol. 23, no. 3, pp. 371-379, 2002.

[4] L. Wang and Y. Zhao, "Large amplitude motion mechanism and non-planar vibration character of stay cables subject to the support motions," Journal of Sound and Vibration, vol. 327, no. 1-2, pp. 121-133, 2009.

[5] M. Spagnuolo and U. Andreaus, "A targeted review on large deformations of planar elastic beams: extensibility, distributed loads, buckling and post-buckling," Mathematics and Mechanics of Solids, pp. 1-23, 2018.

[6] M. P. Omran, A. Amani, and H. G. Lemu, "Analytical approximation of nonlinear vibration of string with large amplitudes," Journal of Mechanical Science and Technology, vol. 27, no. 4, pp. 981-986, 2013.

[7] Z. L. Wang, P. Poncharal, and W. A. De Heer, "Measuring physical and mechanical properties of individual carbon nanotubes by in situ TEM," Journal of Physics and Chemistry of Solids, vol. 61, no. 7, pp. 1025-1030, 2000.

[8] Z. L. Wang, R. P. Gao, P. Poncharal, W. A. De Heer, Z. R. Dai, and Z. W. Pan, "Mechanical and electrostatic properties of carbon nanotubes and nanowires," Materials Science and Engineering C: Materials for Biological Applications, vol. 16, no. 1-2, pp. 3-10, 2001.

[9] Q. Zheng and Q. Jiang, "Multiwalled Carbon Nanotubes as Gigahertz Oscillators," Physical Review Letters, vol. 88, no. 4, 2002.

[10] M. Hemmatnezhad, R. Ansari, and G. H. Rahimi, "Largeamplitude free vibrations of functionally graded beams by means of a finite element formulation," Applied Mathematical Modelling: Simulation and Computation for Engineering and Environmental Systems, vol. 37, no. 18-19, pp. 8495-8504, 2013.

[11] J. B. Gunda, R. K. Gupta, G. Ranga Janardhan, and G. Venkateswara Rao, "Large amplitude vibration analysis of composite beams: Simple closed-form solutions," Composite Structures, vol. 93, no. 2, pp. 870-879, 2011.

[12] A. Nikkar, S. Bagheri, and M. Saravi, "Dynamic model of large amplitude vibration of a uniform cantilever beam carrying an intermediate lumped mass and rotary inertia," Latin American Journal of Solids and Structures, vol. 11, no. 2, pp. 320-329, 2014.

[13] Y. P. Yu, B. S. Wu, Y. H. Sun, and L. Zang, "Analytical approximate solutions to large amplitude vibration of a spring-hinged beam," Meccanica, vol. 48, no. 10, pp. 2569-2575, 2013.

[14] K. K. Raju and G. V. Rao, "Towards improved evaluation of large amplitude free-vibration behaviour of uniform beams using multi-term admissible functions," Journal of Sound and Vibration, vol. 282, no. 3-5, pp. 1238-1246, 2005.

[15] J.-H. He, "Variational approach for nonlinear oscillators," Chaos, Solitons and Fractals, vol. 34, no. 5, pp. 1430-1439, 2007.

[16] S. H. Hoseini, T. Pirbodaghi, M. T. Ahmadian, and G. H. Farrahi, "On the large amplitude free vibrations of tapered beams: 
an analytical approach," Mechanics Research Communications, vol. 36, no. 8, pp. 892-897, 2009.

[17] B. Gheshlaghi, "Large-amplitude vibrations of nanowires with dissipative surface stress effects," Acta Mechanica, vol. 224, no. 7, pp. 1329-1334, 2013.

[18] S. Souayeh and N. Kacem, "Computational models for large amplitude nonlinear vibrations of electrostatically actuated carbon nanotube-based mass sensors," Sensors and Actuators A: Physical, vol. 208, pp. 10-20, 2014.

[19] M. Şimşek, "Large amplitude free vibration of nanobeams with various boundary conditions based on the nonlocal elasticity theory," Composites Part B: Engineering, vol. 56, pp. 621-628, 2014.

[20] R. Nazemnezhad and S. Hosseini-Hashemi, "Exact solution for large amplitude flexural vibration of nanobeams using nonlocal Euler-Bernoulli theory," Journal of Theoretical and Applied Mechanics (Poland), vol. 55, no. 2, pp. 649-658, 2017.

[21] K. Nakamura, K. Kakihara, M. Kawakami, and S. Ueha, "Measuring vibration characteristics at large amplitude region of materials for high power ultrasonic vibration system," Ultrasonics, vol. 38, no. 1, pp. 122-126, 2000.

[22] A. Mozuras and E. Podzharov, "Measurement of large harmonic vibration amplitudes," Journal of Sound and Vibration, vol. 271, no. 3-5, pp. 985-998, 2004.

[23] J. C. Simo, "A finite strain beam formulation. The three-dimensional dynamic problem. Part I," Computer Methods Applied Mechanics and Engineering, vol. 49, no. 1, pp. 55-70, 1985.

[24] P.-F. Pai and A.-H. Nayfeh, "A new method for the modeling of geometric nonlinearities in structures," Computers \& Strucrures, vol. 53, no. 4, pp. 877-895, 1994.

[25] E. Babilio and S. Lenci, "On the notion of curvature and its mechanical meaning in a geometrically exact plane beam theory," International Journal of Mechanical Sciences, vol. 128129, pp. 277-293, 2017.

[26] S. Lenci, F. Clementi, and G. Rega, "Comparing Nonlinear Free Vibrations of Timoshenko Beams with Mechanical or Geometric Curvature Definition," Procedia IUTAM, vol. 20, pp. 3441, 2017.

[27] O. Kopmaz and Ö. Gündoğdu, "On the curvature of an EulerBernoulli beam," International Journal of Mechanical Engineering Education, vol. 31, no. 2, pp. 132-142, 2003.

[28] C. Semler, G. X. Li, and M. P. Païdoussis, "The non-linear equations of motion of pipes conveying fluid," Journal of Sound and Vibration, vol. 169, no. 5, pp. 577-599, 1994.

[29] D. Zhao, J. Liu, and L. Wang, "Nonlinear free vibration of a cantilever nanobeam with surface effects: Semi-analytical solutions," International Journal of Mechanical Sciences, vol. 113, pp. 184-195, 2016.

[30] N. Vlajic, T. Fitzgerald, V. Nguyen, and B. Balachandran, "Geometrically exact planar beams with initial pre-stress and large curvature: Static configurations, natural frequencies, and mode shapes," International Journal of Solids and Structures, vol. 51, no. 19-20, pp. 3361-3371, 2014.

[31] A. H. Nayfeh and D. T. Mook, Nonlinear Oscillations, John Wiley \& Sons, New York, NY, USA, 1979.

[32] F. Benedettini and G. Rega, "Non-linear dynamics of an elastic cable under planar excitation," International Journal of NonLinear Mechanics, vol. 22, no. 6, pp. 497-509, 1987.

[33] H. Koivurova, "The numerical study of the nonlinear dynamics of a light, axially moving string," Journal of Sound and Vibration, vol. 320, no. 1-2, pp. 373-385, 2009.
[34] A. H. Nayfeh, The Method of Normal Forms, Wiley-VCH, Weinheim, Germany, 2nd edition, 2011.

[35] A. Y. Leung and Q. C. Zhang, "Complex normal form for strongly non-linear vibration systems exemplified by Duffingvan der Pol equation," Journal of Sound and Vibration, vol. 213, no. 5, pp. 907-914, 1998.

[36] W. Wang, Q. C. Zhang, and X. J. Wang, "Application of the undetermined fundamental frequency method for analyzing the critical value of chaos," Acta Physica Sinica, vol. 58, no. 8, pp. 5162-5168, 2009. 


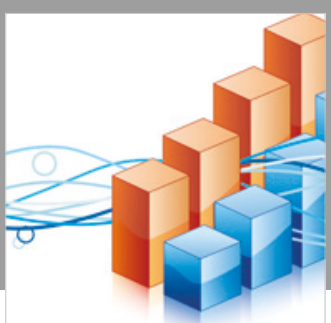

Advances in

Operations Research

\section{-n-m}
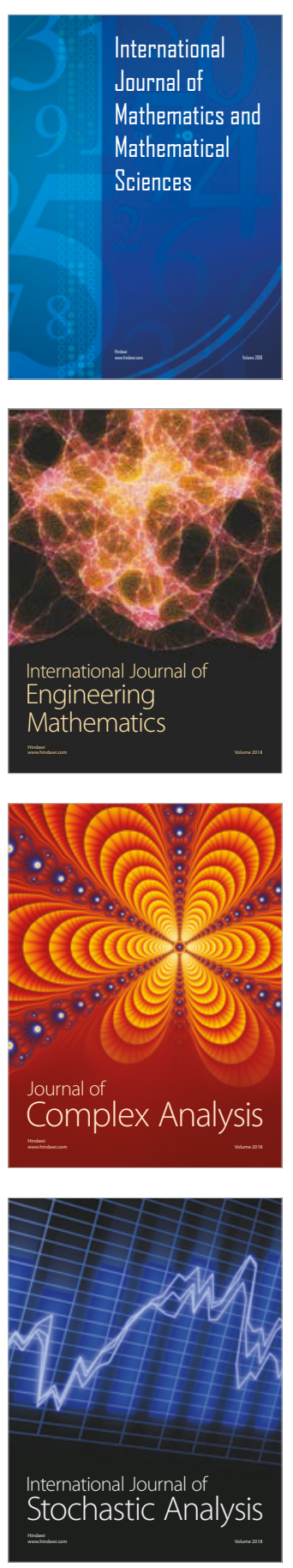
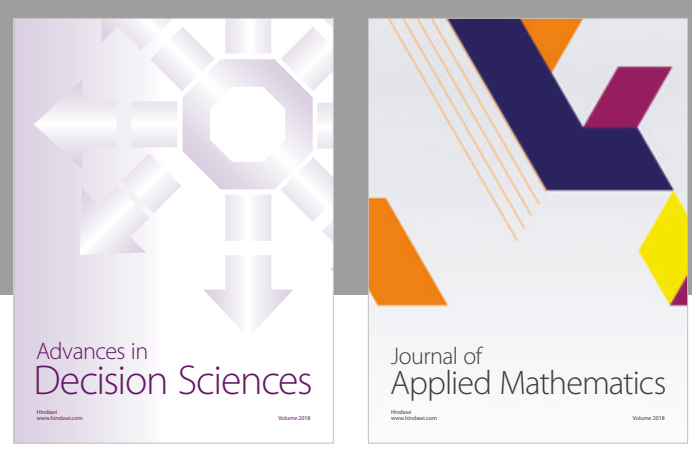

Journal of

Applied Mathematics
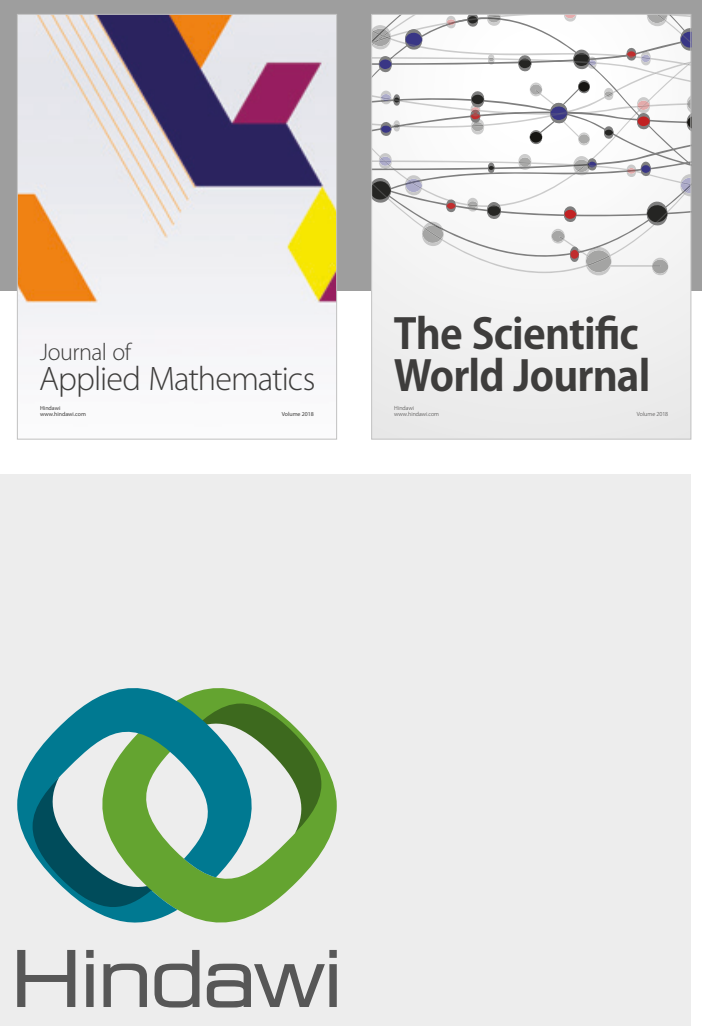

Submit your manuscripts at

www.hindawi.com

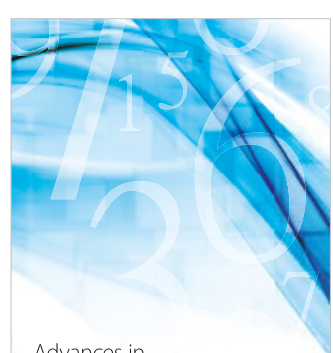

Advances in
Numerical Analysis
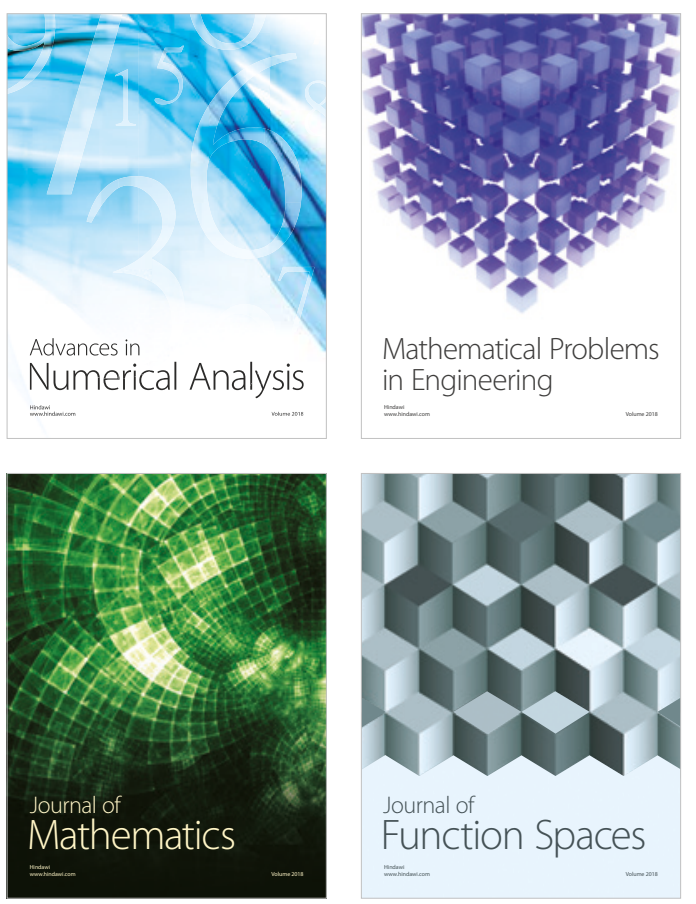

Mathematical Problems in Engineering

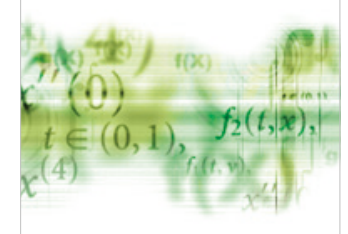

International Journal of

Differential Equations

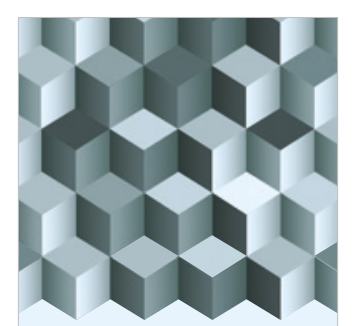

Journal of

Function Spaces

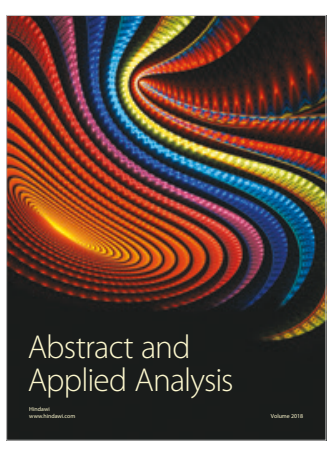

The Scientific

World Journal

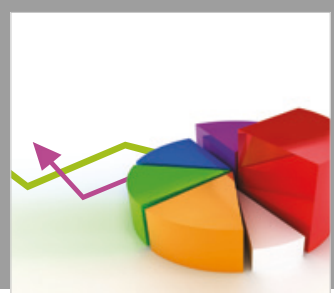

Journal of

Probability and Statistics
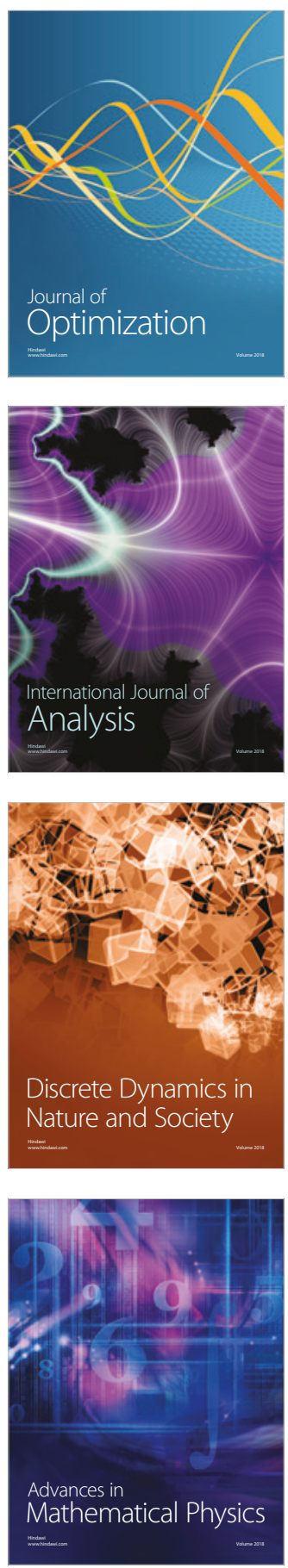